\title{
BRDF Estimations and Normalizations of Sentinel 2 Level 2 Data Using a Kalman-Filtering Approach and Comparisons with RadCalNet Measurements
}

\author{
Bertrand Saulquin
}

check for updates

Citation: Saulquin, B. BRDF Estimations and Normalizations of Sentinel 2 Level 2 Data Using a Kalman-Filtering Approach and Comparisons with RadCalNet Measurements. Remote Sens. 2021, 13, 3373. https://doi.org/10.3390/ rs13173373

Academic Editors: Piero Toscano and Nguyen-Thanh Son

Received: 29 June 2021

Accepted: 17 August 2021

Published: 25 August 2021

Publisher's Note: MDPI stays neutral with regard to jurisdictional claims in published maps and institutional affiliations.

Copyright: (C) 2021 by the author. Licensee MDPI, Basel, Switzerland. This article is an open access article distributed under the terms and conditions of the Creative Commons Attribution (CC BY) license (https:/ / creativecommons.org/licenses/by/ $4.0 /)$.
CS, 5 rue Brindejonc des Moulinais, 31500 Toulouse, France; bertrand.saulquin@cs-group.eu

\begin{abstract}
BRDF estimation aims to characterize the anisotropic behaviour of the observed surface, which is directly related to the type of surface. BRDF theoretical models are then used in the normalization of the satellite-derived observations to virtually replace the sensor at the nadir. Such normalization reinforces the homogeneity within and between satellite-derived time series. Nevertheless, the inversion of the necessary BRDF parameters for the normalization requires the implementation of robust methods to account for the noise in the Level 2 surface reflectances caused by the atmospheric correction process. Here, we compare normalized reflectances obtained with a Kalman filtering approach with $\mathrm{i} /$ the classical weighted linear inversion and ii/a normalization performed using the coefficients of the NASA-MODIS BRDF MCD43A1 band 2 product. We show, using the RadCalNet nadir-view reflectances, that the Kalman filtering approach is a more suitable approach for the Sen2Cor level 2 and the selected sites. Using the proposed approach, daily global maps of land surface BRDF coefficients and the derived normalized Sentinel 2 reflectances would be extremely useful to the global and regional climate modelling communities and for the world's cover monitoring.
\end{abstract}

Keywords: BRDF; normalization; Kalman-filtering

\section{Introduction}

As part of the Copernicus program of the European Commission (EC), the European Space Agency (ESA) has developed and currently operates the Sentinel-2 (S2) optical missions, currently designed as a constellation of two satellites to improve revisit and global coverage from 10 to $60 \mathrm{~m}$ resolution, depending on the spectral bands [1]. Today, the $\mathrm{S} 2 \mathrm{mission}$ is the most commonly used mission among the S2 missions [2]. From Level 0 to Level 2, many complex models are involved in the retrieval of from Top Of Atmosphere (TOA) to Bottom Of Atmosphere (BOA) signals [1]. Validation of the Level 1 TOA [3] and the Level 2 BOA estimates [3] is a major topic when ensuring that the activities of the mission address the initial requirements. Such validation processes involve in-situ data or Fiducial Reference Measurements (FRM) [4] that represent "truths" to be targeted by the Level 1 or Level 2 products studied. The Mission Performance Centre (MPC), as part of the European Space Agency's S2 ground segment, operationally carries out the routine S2 calibration and validation activities and investigates potential tracks for improvement, as explored in this paper.

At present, the ESA's official S2 level 2 surface reflectances are delivered by the Sen2Cor processor [5]. Sen2cor's surface reflectances are currently not normalized, i.e., they are still in the sensor-viewing geometry. The context of the European Copernicus Program requires a reliable and comparable long-term time series of surface products that are acquired using different sensors and conditions of acquisition. The international community also works to harmonize the acquisitions between sensors, and, among them, the CEOS Analysis Ready Data for Land (CARD4L) proposes specifications to be shared between data producers for harmonizing the Level 2 Surface Reflectance [6]. 
More specifically, the goal here is to propose a robust method to estimate the Bidirectional Reflectance Distribution Function (BRDF) from Sen2Cor reflectances, which will be used for the normalization to ensure a more consistent time series. The Kalman Filter (KF) presented in this study is a similar technique to the assimilation [7], one that is particularly dedicated to the estimation of parameters in noisy datasets [8]. This method, which is widely used for a large number of applications, consists of recursively updating the model variables each time new observations are available. In the absence of new observations, typically for the cloudy conditions often observed in optical sensors such as S2, the KF state vector is propagated using a time-evolving model.

In this paper, we examine the BRDF parameter estimations using two methods, a classical weighted linear inversion as implemented by NASA for the Moderate Resolution Imaging Spectroradiometer mission (MODIS) [9] and the Kalman filtering approach. After the BRDF parameter estimation, the Sen2Cor reflectances are normalized, i.e., estimated for the sensor position at (here) the nadir using the estimated BRDF coefficients. To complement these two approaches, we use the MCD43A1 BRDF parameters estimated for the Band 2 of MODIS [10]. The MODIS Band 2 is spectrally close to the $\mathrm{S} 2$ band B8A, and can thus be used for normalizing $\mathrm{S} 2$ data for this band, assuming that the surface is homogeneous over the $500 \mathrm{~m}$ resolution MODIS pixel used. We then validate the tested models against the in-situ RadCalNet data [11]. Our approach is motivated by our ultimate objective which is to derive robust BRDF coefficients and normalized reflectances from noisy observations and minimize the biases and the uncertainties compared with the RadCalNet reference validation dataset.

\section{Materials and Methods}

\subsection{Satellite Level 2 Products Using Sen2Cor 2.09}

Sen2Cor is the Level 2 processor provided by ESA to correct Level 1 from the atmospheric signal $[12,13]$. The outputs of the Sen2Cor processor are surface reflectances, aerosol optical thicknesses at $550 \mathrm{~nm}$ (AOT550) and water vapor (WV) concentrations. The atmospheric correction is performed using a set of Look-Up tables generated via libRadtran [14]. In summary, some LUTs model the atmospheric signal. AOT550 is computed using the Dark \& Dense Vegetation assumption (DDV, [15]), i.e., a spectral model that uses, in this case, B04 and B12. In the atmospheric correction process, firstly, the signal is corrected from the gaseous transmittances, $T_{g a z}(\lambda)$. Then, from the AOT550, the atmospheric total (diffuse + direct) transmittances $T_{\text {up }}(\lambda) \& T_{\text {down }}(\lambda)$ are computed and the surface reflectance $\rho_{\text {surf }}(\lambda)$ is obtained using the following observation model (the term to be estimated is in bold, i.e., the spectral surface reflectances):

$$
\rho_{T O A}(\lambda)=\left[\rho_{\text {path }}(\lambda)+\frac{\rho(\lambda)}{1-S_{\text {atm }}(\lambda) \boldsymbol{\rho}(\lambda)} * T_{\text {up }}(\lambda) * T_{\text {down }}(\lambda)\right] * T_{g a z}(\lambda)
$$

In Equation (1), $S_{\text {atm }}(\lambda)$ is the spherical albedo. $\rho_{\text {path }}(\lambda)$ is the combined Rayleigh and aerosol reflectances. In Sen2Cor, the used pressure is proportional to the altitude [12]. As the surface BRDF is not modelled in the used LUT by Sen2Cor, to uncouple the surface from the atmospheric signal [16], an isotropic assumption of the surface signal is performed, i.e., $\mathrm{L}_{\text {surf }}(\lambda)=\rho_{\text {surf }}(\lambda) / \pi$ [12]. The Sen2Cor processor requires that auxiliary files [5] and a Digital Elevation Model (DEM) be installed (SRTM [17]). More details on the Sen2Cor processor are provided in [5].

The level 2 time series are processed at $20 \mathrm{~m}$ resolution using the Level 1 tiles that were downloaded from the ESA facilities and the Sen2Cor processor v2.09. A $3 * 3$ pixel box is considered for the matchups to cover the surface observed in-situ by the sensor. Cloud and cloud shadows are filtered, and the satellite minus in-situ time difference is taken to be lower than $30 \mathrm{~min}$.

We consider here both the S2A and S2B datasets from 2016 to 2020 without differentiation, as the geometry of acquisition (relative orbits) for S2A and S2B is the same. It also allows for more observations in time for the BRDF parameter estimations. 


\subsection{In-situ Surface Reflectances from the RadCalNet Network}

RadCalNet dataset provides in-situ surface reflectances at nadir over a spectral range from $400 \mathrm{~nm}$ to $1000 \mathrm{~nm}$ (and up to $2500 \mathrm{~nm}$, depending on each site's instrumentation) at $10 \mathrm{~nm}$ spectral resolution [11]. These datasets are used intensively for satellite sensor validation [18]. Three RadCalNet sites are used in this study: the La Crau site in France, the Gobabeb site in Namibia and the Railroad Valley site in the US. The La Crau site shows moderate vegetation (grass) during winter and spring, while it tends towards semi-arid soil surfaces in summer. Gobabeb and Railroad Valley are typical flat deserts, bright targets homogeneous in time. RadCalNet in-situ measurements are derived from a Cimel CET312 photometer at La Crau and Gobabeb that can target either the sky or the surface to derive the atmospheric components and the surface BRDF [11]. At Railroad Valley, a ground-viewing radiometer (GVRs) with 8 bands is used [19]. The surface reflectances for the RadCalNet sites are normalized for nadir view using the estimated local BRDF model derived with the in-situ data.

Figure 1 shows the geometry of acquisitions for the S2 A\&B over the three RadCalNet sites studied. In Figure 1, thetas is the sun zenith angle, thetav is the viewing angle and $\mathrm{dphi}$ is the relative azimuth angle (see [20] for the definition of the angles). At Gobabeb and La Crau, the S2 A\&B viewing angles are low, with a maximum of $4^{\circ}$ and $7.5^{\circ}$ respectively (Figure 1, in green), making the normalization process less important than for other sensors with larger viewing angles.
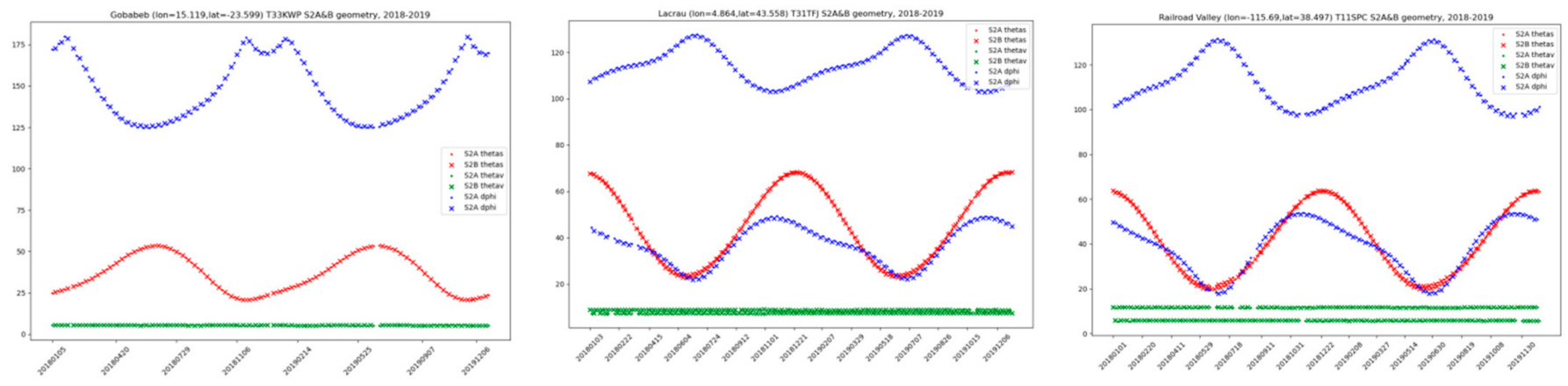

Figure 1. Geometry of acquisition for the S2A\&B at the three selected RadCalNet sites. Left, Gobabeb is observed only on the orbit 107. Middle, La Crau is observed on both orbits 8 and 108. Right, Railroad Valley is observed on orbits 27 and 127.

At Railroad Valley, the viewing angle reaches $11.4^{\circ}$ for the orbit 127 . Among the in-situ data available from RadCalNet, we use the normalized (nadir viewing) surface reflectance

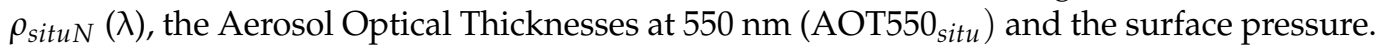

\subsection{BRDF Models and Reflectance Normalization}

The normalization of the surface reflectances requires the use of a BRDF model describing the surface reflectance anisotropy. It is now commonly admitted that the BRDF can be represented with good accuracy by using a kernel-based semi-empirical model, which describes the reflectance as a sum of three angular kernels, Ki, weighted by the coefficients $F i$ :

$$
\operatorname{BRDF}\left(\theta_{s}, \theta_{v}, \Delta \varphi, \lambda\right)\left(s r^{-1}\right)=\frac{L u}{E d}=\sum_{k=0}^{2} F_{k}(\lambda) * K_{k}\left(\theta_{s}, \theta_{v}, d \varphi\right)
$$

In Equation (2). $\theta_{s}, \theta_{v}, \Delta \varphi$ are, respectively, the sun, the view and the relative azimuth angles. $\lambda$ is the wavelength. Lu is the upward directional radiance and Ed the irradiance, i.e., the angular integration of the downward luminance Ld over the downward hemisphere (in the case, as for Sen2Cor, of a plan parallel atmosphere model [21]).

For this study, we use the initial BRDF model of Roujean et al. (2002) and the current RossTick-LiSparse (Ross-Li) model used by NASA [22] and [9]. In Equation (2), F0 repre- 
sents the isotropic part of the reflectance, F1 mimics the contribution of the geometrical effects and F2 stands for the contribution of multiple scatterings.

\section{Nadir-Normalized S2 Reflectances}

Normalized reflectances are computed using the estimated BRDF to virtually replace the sensor at nadir. The nadir-normalized reflectances are then directly comparable with the RadCalNet nadir reflectance measurements. The goal of such normalization is also to favour inter-comparisons within and between the satellite time-series that are acquired within different geometric conditions. Finally, with these BRDF parameters, we apply the following equation to derive the nadir-normalized reflectances [23,24].

$$
\rho_{N}(\lambda)=\rho\left(\lambda, \theta_{s}, \theta_{v}, \Delta \varphi\right) \frac{B R D F\left(\theta_{s}=\theta_{s}, \theta_{v}=0, \Delta \varphi=0\right)}{\operatorname{BRDF}\left(\theta_{s}, \theta_{v}, \Delta \varphi\right)}
$$

\subsection{BRDF Parameter Estimation Using the Kalman Filter}

The Kalman Filter (KF) is a recursive two-step algorithm that provides an estimate, at each time step $n$, of a state variable, $X n$, of a system. It has been designed primarily to monitor processes for which it is crucial to accurately estimate the state of the system in the presence of noisy observations. In the KF, the estimation of the state variable $X$ at time step $n-1$ is first propagated in time by a linear temporal state-transition model, namely, the operator $F$, to obtain the a priori estimate $X_{n}^{a}$ for time step $n$ :

$$
X_{n}^{a}=F \cdot X_{n-1}
$$

The state vector covariance matrix $C$, representing the uncertainty on $X$, is propagated using the equation:

$$
C_{n}^{a}=F \cdot C_{n-1} \cdot F_{T}+Q
$$

$Q$ stands for the model noise variance, i.e., the uncertainty of the model compared to the real physical process.

In a second step, measurements (here, the surface reflectances) $Z$ are related to the state variable by the linear relationship $Z=H . X+v$, where $H$ is the observation model (matrix), and $v$ a random white noise of variance $R$ describing the measurement noise. The state vector estimate and covariance are then corrected using:

$$
X_{n}=X_{n}^{a}+G_{n}\left(Z_{n}-H \cdot X_{n}^{a}\right) ; C_{n}=\left(1-G_{n} \cdot H_{n}\right) \cdot C_{n}^{a}
$$

where $G_{n}$ is the Kalman gain expressed as:

$$
G_{n}=C_{n}^{a} H^{T}\left(H C_{n}^{a} H_{T}+R\right)^{-1}
$$

The Kalman gain is thus a function of the state vector covariance and the measurement noise. In the present study, the state vector $X$ is the set of parameters of the BRDF model, i.e., $[k 0, k 1, k 2]$. The observation matrix for the reflectance is then simply written as $H=[1 f 1 f 2]$.

\subsubsection{Outlier Removals}

One may use the first step of the Kalman filtering to verify if an observation Z, newly acquired at $d t$, the time step between the new and the latest observation, follows the $\mathrm{KF}$ hypothesis. The advantage of the KF, compared to a simple linear regression, is the possibility of detecting the outliers, given the a priori knowledge of the a priori state vector. To ensure the KF hypothesis, the new observation $\mathrm{Z}$ must ensure:

$$
\left|Z-H \cdot X_{n}^{a}\right|^{2} \leq 4 * \operatorname{diag}\left(R+H \cdot C_{n}^{a} \cdot H^{T}\right)
$$




\subsubsection{Temporal Model}

Temporal model of the model noise variance matrix $Q$ is proportional to the state vector, with the off-diagonal elements set to zero. We introduced some variance regarding $\mathrm{dt}$, as the more the gap between the observations increases, the less the latest estimation of $\mathrm{X}$ is relevant:

$$
\operatorname{diag}(Q)=\left|X_{n-1}\right| 10^{-3}-10^{-4} * \mathrm{dt}
$$

\subsubsection{Observation Uncertainties}

Observation uncertainty covariance matrix $\mathrm{R}_{i}$ for observation $\mathrm{i}$ is set to be proportional to the reflectance level:

$$
\mathrm{R}_{i}(\lambda)=\left(0.05 \rho_{i}(\lambda)+R_{0}\right)^{2}
$$

where $R_{0}$ is set to $3 \%$ of the standard deviation of $\rho(\lambda)$, the surface reflectance.

\subsection{BRDF Parameter Estimation Using the Standard Weighted Linear Inversion}

The results obtained with the KF are compared to the method used by NASA and MODIS [9], i.e., a standard least square, optimized here using a Bounded Variable Least Squares (BVLS) regression analysis to avoid geophysically meaningless estimates. The method consists of inverting the Equation (2) with the data collected over a sliding temporal window of 30 days. To favor those observations which are temporally close to the studied date, a temporal weighting is performed to give more importance to the data close to the window's center [9].

\subsection{Aerosol and Rayleigh Corrected Sen2Cor Reflectances}

The angular kernels $\mathrm{K}$ are, by definition, very sensitive to the geometry. In order to unmix the errors performed by the atmospheric corrections from the BRDF estimation \& the reflectance normalization, and before the BRDF estimation, we apply an aerosol and Rayleigh correction, given the aerosol loads and the surface pressure measured at the RadCalNet stations. We next use the libRadtran libraries to correct the Sen2Cor estimates, given the in-situ observations. With $\rho_{\text {path }}$ being the aerosol and Rayleigh reflectances (including the coupling term [25] between the Rayleigh and aerosols), the error $E \rho_{\text {path }}$ is estimated using:

$$
E \rho_{\text {path }}=\rho_{\text {path }} \text { S2C }-\rho_{\text {path_situ }}
$$

and the transmittance error is estimated using:

$$
E T d_{\text {path }}=T d_{\text {path }}{ }_{S 2 C} / T d_{\text {path_situ }}
$$

In the equation above, $\rho_{\text {path }}$ S2C (and respectively $T d_{\text {path }}$ S2C ) is the path reflectance estimated using the AOT, aerosol model and surface pressure used by Sen2Cor, while $E \rho_{\text {path }} h_{\text {situ }}$ is the path reflectance estimated using the measurements at the RadCalNet stations.

Finally, the corrected Sen2Cor surface reflectances are estimated using Equation (1):

$$
\rho^{*}(\lambda)=\rho(\lambda) * E T d_{\text {path }}+\frac{E \rho_{\text {path }}}{T d_{\text {path_situ }}}
$$

It must be noted that this preliminary correction is not mandatory for BRDF estimation and reflectance normalization. Nevertheless, the normalized reflectances after this step showed better results compared with the in-situ data, exhibiting the importance of this correction.

\subsection{Statistical Diagnostics}

Qualification of the results is performed using accuracy A and uncertainties $U$ of the estimated normalized reflectances:

$$
\mathrm{A}\left(\hat{\rho}^{*}(\lambda)\right)=\frac{1}{\mathrm{n}} \sum\left(\hat{\rho}^{*}(\lambda)-\rho^{*}(\lambda)\right)
$$


and

$$
\mathrm{U}\left(\hat{\rho}^{*}(\lambda)\right)=\sqrt{\frac{1}{\mathrm{n}} \sum\left(\hat{\rho}^{*}(\lambda)-\rho^{*}(\lambda)\right)^{2}}
$$

\section{Results}

\subsection{Estimated BRDF Coefficients}

Figure 2 shows the estimated BRDF coefficients at the three RadCalNet stations using the KF and the Roujean Kernels. At Gobabeb, the coefficients are constants in time, while, at $\mathrm{La} \mathrm{Crau}$, some seasonal cycles appear. The magnitude and the uncertainties of the estimated coefficients are comparable to those obtained by Samain and Roujean [26] for Bare Lands (Gobabeb-like) and semi-arid open lands, using a similar approach and the SPOT-4 vegetation instrument. At Railroad Valley, a possible scenario to explain the increase in the volume scattering coefficient is an increase in the surface roughness due to a recrudescence of dusts [24].
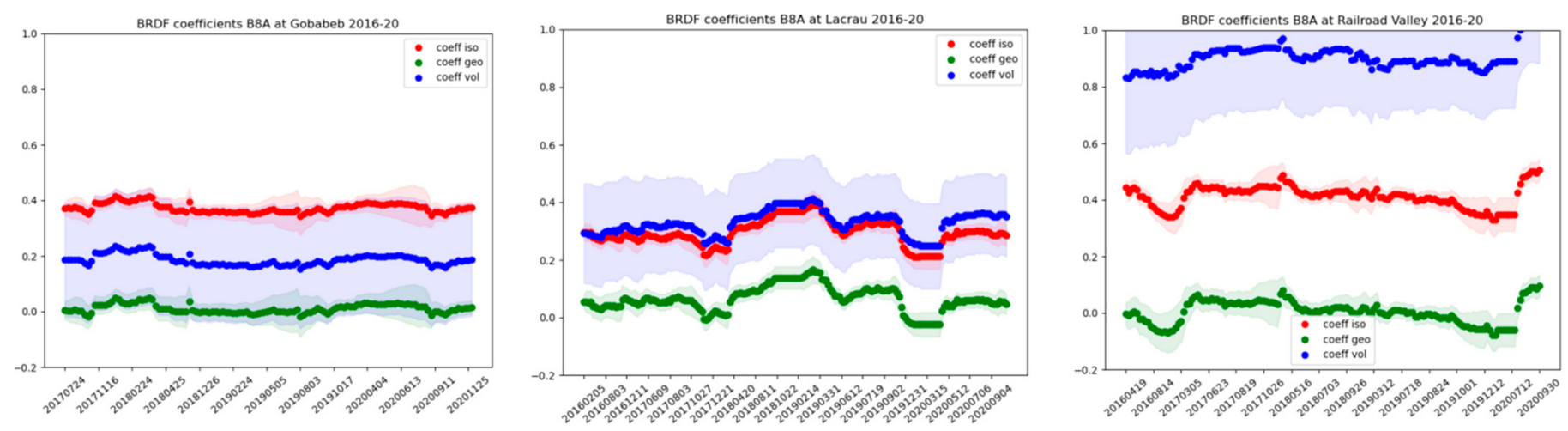

Figure 2. Time series of BRDF cefficients using the Roujean Kernels and the Kalman filtering approach, and related uncertainties for each parameter.

\subsection{Normalized Sen2cor Surface Reflectances Using the Roujean Kernels}

In Figure 3 the normalization results are shown in accuracy (biases) and uncertainties for the three RadCalNet sites. At Gobabeb the normalization process tends to minimize the bias. The KF approach shows less bias than the classical linear inversion with the temporal weighting. Uncertainty reductions at Gobabeb are very low because of the very low viewing angle $\left(<4.5^{\circ}\right)$ and the isotropic behavior of the surface (Figure 2).

At $\mathrm{LaCrau}$, the aerosol and the Rayleigh correction introduce a minimal bias of $2 \%$ for $\mathrm{B} 01$ and B02, but they reduce the uncertainties at these bands. At La Crau, the KF reduces uncertainties more than the classical linear inversion, with a mean reduction of $2 \%$ for all considered bands.

At Railroad Valley, the initial Sen2Cor products show positive biases from 10 to $20 \%$, depending on the bands. Aerosol and Rayleigh corrections do not correct the observed biases (Figure 3, right). The KF normalization diminishes the uncertainties by $5 \%$, which is the larger of the reductions observed among the three sites. This important improvement in the time series may be due to the fact that the viewing angle for orbit 127 is larger at Railroad Valley, and the surface here is more anisotropic compared with Gobabeb (Figure 2).

The red stars in Figure 3 show the normalization results obtained using the MODIS BRDF MCD43A1 (500 m resolution) BRDF parameters for the MODIS band 2, spectrally close to the S2 B08, applied using the Ross-Li kernels and Equation (3). S2-normalized reflectances at Gobabeb using these coefficients show some slight positive biases of 5\% and are in agreement with our estimates at La Crau and Railroad Valley. The results for uncertainties are worse when compared with our approach, with some uncertainty levels of 3, 11 and 19\%, respectively, for Gobabeb, La Crau and Railroad Valley. These results 
show that our approach performs better for the S2 B08 than the direct application of the MODIS-estimated BRDF parameters.
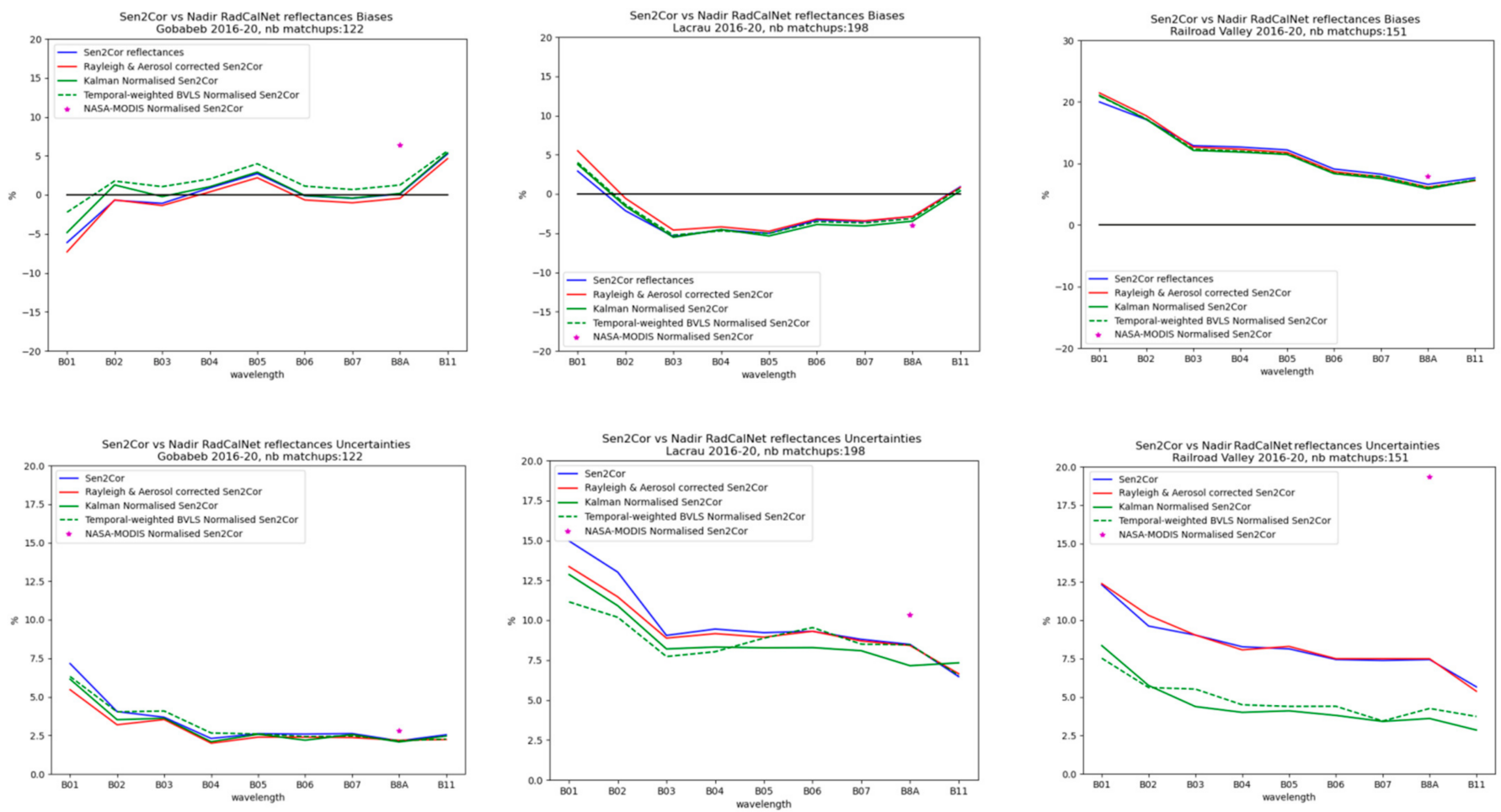

Figure 3. Statistical assessment of normalized Sen2Cor reflectances vs. in-situ data using the Roujean Kernels. Top, accuracy (biases) and bottom, uncertainties. The red stars correspond to the normalization results obtained using directly the MODIS band 2 BRDF MCD43A1 (500 m resolution) parameters and the related Ross-Li kernels.

\subsection{Normalized Sen2cor Surface Reflectances Using the Ross-Li Kernels at Railroad Valley}

In Figure 4, the normalization results using the Ross-Li Kernels for the Railroad Valley site are shown. Both the weighted linear inversion and the KF introduce some positive biases, and the KF approach shows lower bias than the classical weighted linear inversion. With the exception of B01, uncertainty reductions are larger using the KF filtering compared to the weighted linear inversion. Note that, for the La Crau and Gobabeb sites, the results (not shown) also clearly showed lower performances compared with the Roujean kernels.
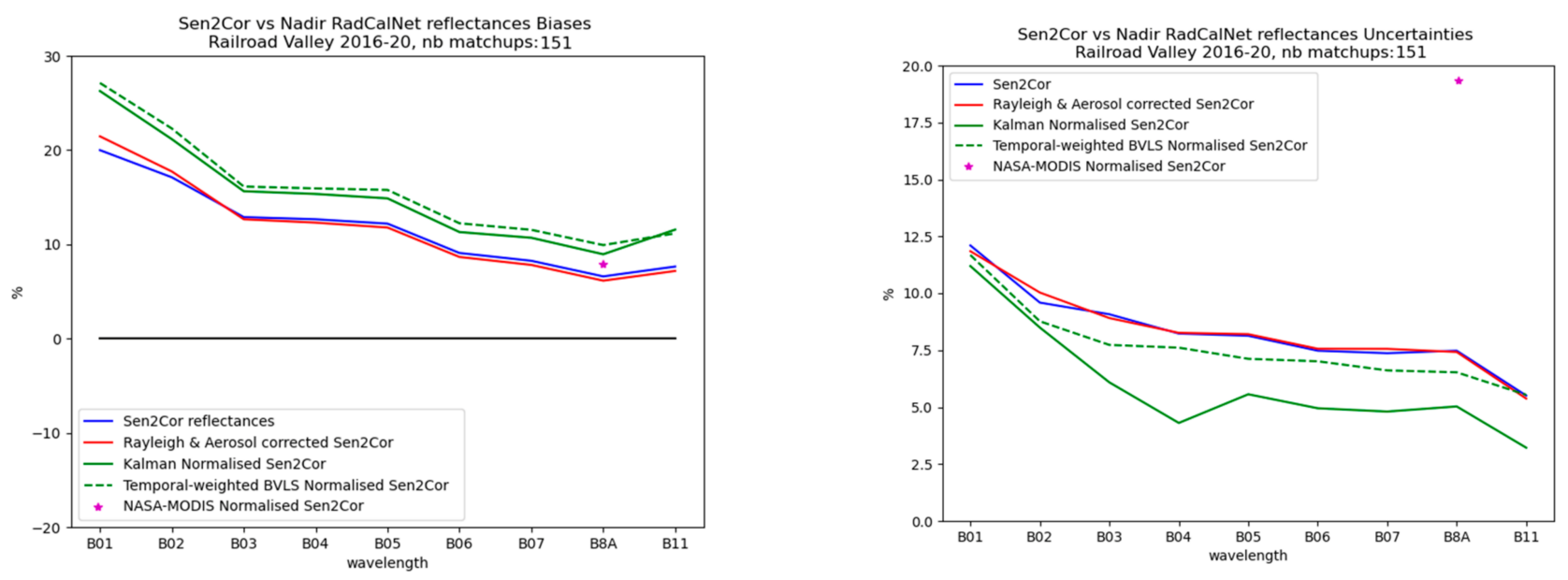

Figure 4. Statistical assessment of normalized Sen2Cor reflectances vs. in-situ data using the Ross-Li kernels at Railroad Valley. Left, accuracy (biases) and right, uncertainties. The red stars correspond to the normalization results obtained using directly the MODIS band 2 BRDF MCD43A1 (500 m res.) parameters and the related Ross-Li kernels. 


\subsection{Spatial Distribution of the Estimated BRDF Coefficients for La Crau and B8A}

Figure 5 shows the estimated coefficients at La Crau for the 20200815 using four images: 20200731, 20200805, 20200807 and 20200815. While the isotropic coefficients are comparable for the KF and the weighted linear inversion (Figure 5, left) it is clear that the geometric and the volume scattering coefficients estimated using the KF are spatially smoother. The linear inversion, even if bounded or constrained (BVLS, [27]), shows spatial discontinuities in the estimations. This is caused by the high sensitivity of the used BRDF model to the noise in the observations. This topic is particularly well addressed by the Kalman filtering approach.

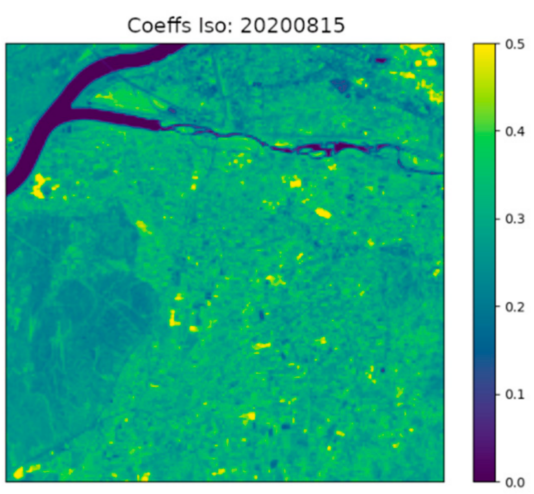

Coeffs Iso: 20200815

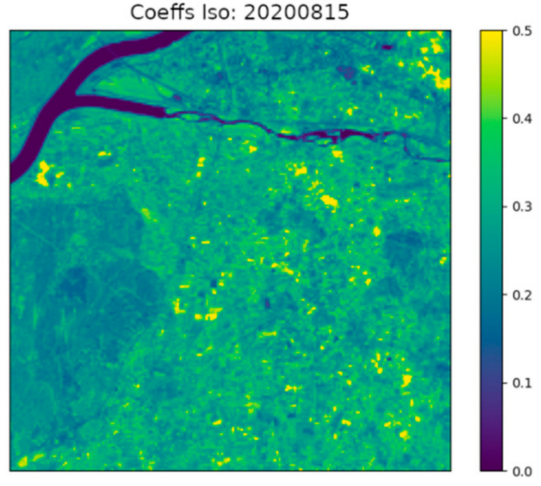

Coeffs Geo: 20200815

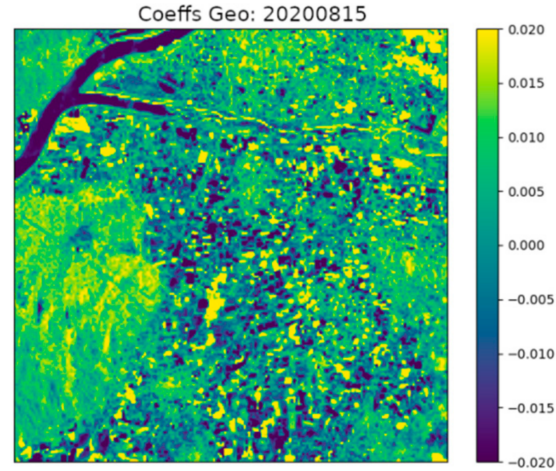

Coeffs Geo: 20200815

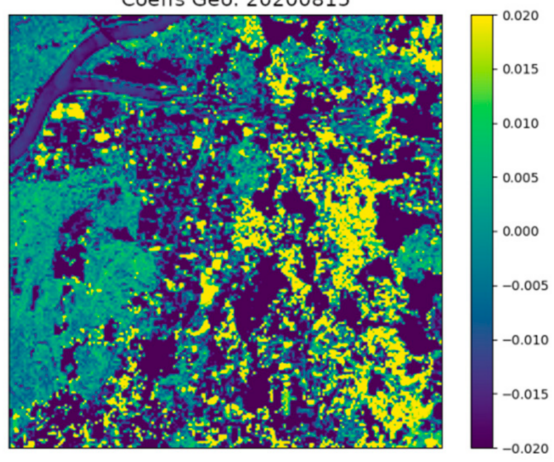

Coeffs Vol: 20200815

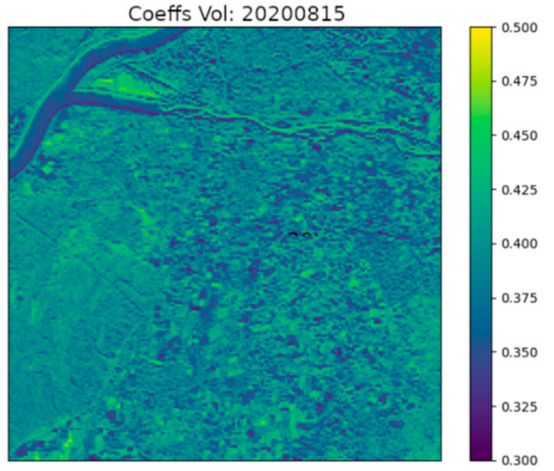

Coeffs Vol: 20200815

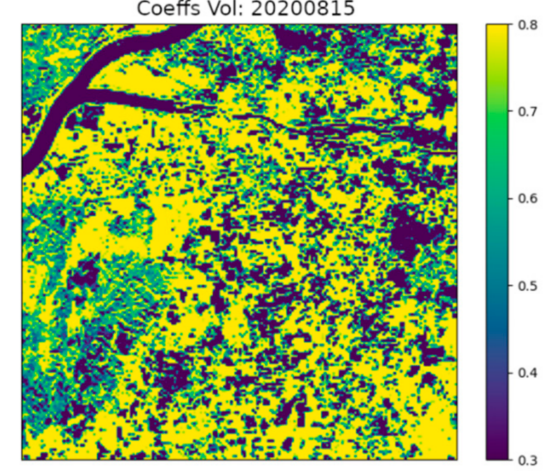

Figure 5. BRDF coefficients k0 (isotropic, top), k1 (geometric, middle) and k2 (volume scattering, right) at La Crau for the S2 B8A image of the 20200815 obtained using the Kalman filter (top) and the classical weighted linear inversion (bottom).

\section{Discussion}

To our knowledge, this study is the first to normalize S2 Sen2cor reflectances using a Kalman filtering approach. Here, we show that the BRDF models used are very sensitive to the noise measurements. The atmospheric correction process performed to retrieve the surface reflectances, used as input of the BRDF estimation, introduces itself some noise. The use of the Kalman filtering, robust to the noise, allows for the retrieval of more consistent and reliable BRDF parameters. Conversely, the use of a classical linear inversion (OLS, or constrained-bounded version BVLS) often leads to spatial and temporal discontinuities in the estimated geometric and volume-scattering coefficients. These inversion issues were more visible when looking at the estimated BRDF coefficients. In the case of a classical linear inversion being used, the BRDF coefficients may not have any geophysical reality. These issues may not appear while checking only the differences between the estimated normalized reflectances and the RadCalNet data. This is caused by i/ non-homogeneous BRDF coefficients in time and space, which may lead to reliable normalized reflectances and ii/ the close to nadir position of the $\mathrm{S} 2$ sensors with, most of the times, a viewing angle lower than $10^{\circ}$. In the case of S2, the simulation of the nadir position of the sensor is by definition often close to the non-normalized estimates. 
In any case, the KF approach showed better results in terms of the biases and uncertainties than the classical weighted linear inversion at the three selected RadCalNet sites of Gobabeb, La Crau and Railroad Valley. Regarding the BRDF kernels that were used, the Roujean kernels performed better than the Ross-Li kernels at the RadCalNet sites. This might be caused by the difference in definition of the volume scattering coefficients between the two models. For other types of surfaces (typically forests) that are not addressed by the RadCalNet sites studied here, these results may change.

Compared with the direct use of the MODIS MCD43A1 (500 m resolution) BRDF parameters, normalization results for bands B08 showed great improvements using the Kalman filtering approach, especially for uncertainties. This stresses the need to estimate dedicated BRDF parameters using the S2 data.

Further improvements to the approach may involve more complex temporal models for the Kalman filtering, introducing, for example, some external classifications. The proposed approach provides currently potential improvements for S2 and the possibility of delivering reliable BRDF coefficients at global scale. The provision of daily global maps of land surface BRDF coefficients and the derived normalized S2 reflectances with such an approach will be extremely useful to the global and regional climate modelling communities and the world cover monitoring. It also allows for potential improvements for other current surface albedo products, either those derived from the geostationary MSG (Meteosat Second Generation) or the sun-synchronous S3 (Sentinel 3), Landsat (Land Remote-Sensing Satellite), MODIS and PACE (Plankton, Aerosol, Cloud, ocean Ecosystem) missions. The approach can also be applied to other related biophysical variables such as the Leaf Area Index (LAI), the fraction of vegetation cover and the fraction of Absorbed Photosynthetically Active Radiation (FAPAR). The proposed work also contributes to the future use of assimilation techniques, typically ECMWF (European Centre for MediumRange Weather Forecasts) products, to enhance the satellite-derived estimates. In a larger vision, any inversion using satellite reflectance observations could take advantage of the KF approach proposed here, including the atmospheric correction itself. In the case of the atmospheric correction that relies on non-linear functions, the Extended Kalman Filter will have to be used [28].

Thus, the implementation of the KF technique in the official processing chains would participate constructively with the European Space Agency's (ESA) Calibration/Validation Strategy to create continuous time series and increase the inter-sensor's interoperability in the optical domain [29].

Funding: This research was funded by the ESA Sentinel-2 Mission Performance Centre (S2MPC).

Institutional Review Board Statement: Not applicable.

Informed Consent Statement: Not applicable.

Data Availability Statement: All Sentinel data used here are available from the ESA scihub facilities.

Acknowledgments: We would like to thank Valentina Boccia, Cal/Val Manager from the S2MPC for supporting the project and the anonymous reviewers whose comments have helped to improve the manuscript.

Conflicts of Interest: The authors declare no conflict of interest. 


\section{References}

1. Drusch, M.; Del Bello, U.; Carlier, S.; Colin, O.; Fernandez, V.; Gascon, F.; Bargellini, P. Sentinel-2: ESA's optical high-resolution mission for GMES operational services. Remote Sens. Environ. 2012, 120, 25-36. [CrossRef]

2. Copernicus Sentinel Data Access Annual Report 2019. Available online: https://sentinels.copernicus.eu/web/sentinel/news/ - / asset_publisher/xR9e/content/copernicus-sentinel-data-access-annual-report-2019; jsessionid=4DC08B0ABC1B60CB9 A889CD1AF2B53B1.jvm2?redirect=https \%3A\%2F\%2Fsentinels.copernicus.eu $\% 2 F w e b \% 2 F s e n t i n e l \% 2 F n e w s \% 3 B j s e s s i o n i d \%$ 3D4DC08B0ABC1B60CB9A889CD1AF2B53B1.jvm2\%3Fp_p_id\%3D101_INSTANCE_xR9e\%26p_p_lifecycle\%3D0\%26p_p_ state\%3Dnormal\%26p_p_mode\%3Dview\%26p_p_col_id\%3Dcolumn-1\%26p_p_col_count\%3D1\%26_101_INSTANCE_xR9 e_keywords\%3D\%26_101_INSTANCE_xR9e_advancedSearch\%3Dfalse\%26_101_INSTANCE_xR9e_delta\%3D20\%26_101 _INSTANCE_xR9e_andOperator\%3Dtrue (accessed on 28 June 2021).

3. S2MPC Team. Sentinel-2 Data Quality Report; Tech. Rep. S2-PDGS-MPC-DQR; 2021. Available online: https://sentinels copernicus.eu/documents/247904/685211/Sentinel-2_L1C_Data_Quality_Report.pdf/6ad66f15-48ca-4e65-b304-59ef00b7f0e0 ?t=1628261039520 (accessed on 28 June 2021).

4. Thorne, P.W.; Diamond, H.J.; Goodison, B.; Harrigan, S.; Hausfather, Z.; Ingleby, N.B.; Jones, P.D.; Lawrimore, J.H.; Lister, D.H.; Merlone, A.; et al. Towards a global land surface climate fiducial reference measurements network. Int. J. Climatol. 2018, 38, 2760-2774. [CrossRef]

5. Level-2A Algorithm Theoretical Basis Document. 2011. Available online: https://earth.esa.int/c/document_library/get_file? folderId=349490\&name=DLFE-4518.pdf (accessed on 28 June 2021).

6. Lewis, A.; Lacey, J.; Mecklenburg, S.; Ross, J.; Siqueira, A.; Killough, B.; Szantoi, Z.; Tadono, T.; Rosenavist, A.; Goryl, P.; et al. CEOS analysis ready data for Land (CARD4L) overview. In Proceedings of the IGARSS 2018-2018 IEEE International Geoscience and Remote Sensing Symposium, Valencia, Spain, 22-27 July 2018; pp. 7407-7410.

7. Talagrand, O. Assimilation of observations, an introduction (gtspecial issueltdata assimilation in meteology and oceanography: Theory and practice). J. Meteorol. Soc. Jpn. 1997, 75, 191-209. [CrossRef]

8. Kalman, R.E. A New Approach to Linear Filtering and Prediction Problems. Trans. ASME J. Basic Eng. 1960, 35-45. [CrossRef]

9. Strahler, A.H.; Muller, J.; Schaaf, C.B.; Hu, B.; Muller, J.P.; Lewis, P.; Barnsley, M.J. MODIS BRDF/albedo product: Algorithm theoretical basis document version 5.0. MODIS Doc. 1999, 23, 42-47.

10. Justice, C.O.; Townshend, J.R.G.; Vermote, E.F.; Masuoka, E.; Wolfe, R.E.; Saleous, N.; Roya, D.P.; Morisette, J.T. An overview of MODIS Land data processing and product status. Remote Sens. Environ. 2002, 83, 3-15. [CrossRef]

11. Bouvet, M.; Thome, K.; Berthelot, B.; Bialek, A.; Czapla-Myers, J.; Fox, N.P.; Goryl, P.; Henry, P.; Ma, L.; Marcq, S.; et al. RadCalNet: A radiometric calibration network for Earth observing imagers operating in the visible to shortwave infrared spectral range. Remote Sens. 2019, 11, 2401. [CrossRef]

12. Louis, J.; Pflug, B.; Main-Knorn, M.; Debaecker, V.; Mueller-Wilm, U.; Iannone, R.Q.; Cadau, E.H.; Boccia, V.; Gascon, F. Sentinel-2 global surface reflectance level-2A product generated with Sen2Cor. In Proceedings of the IGARSS 2019-2019 IEEE International Geoscience and Remote Sensing Symposium, Yokohama, Japan, 28 July-2 August 2019; pp. 8522-8525.

13. Saulquin, B.; Fablet, R.; Bourg, L.; Mercier, G.; d'Andon, O.F. MEETC2: Ocean color atmospheric corrections in coastal complex waters using a Bayesian latent class model and potential for the incoming sentinel 3-OLCI mission. Remote Sens. Environ. 2016, 172, 39-49. [CrossRef]

14. Mayer, B.; Kylling, A. Technical note: The libRadtran software package for radiative transfer calculations-Description and examples of use. Atmos. Chem. Phys. 2005, 5, 1855-1877. [CrossRef]

15. Kaufman, Y.J.; Remer, L.A. Detection of forests using mid-IR reflectance: An application for aerosol studies. IEEE Trans. Geosci. Remote Sens. 1994, 32, 672-683. [CrossRef]

16. Hu, B.; Lucht, W.; Strahler, A.H. The interrelationship of atmospheric correction of reflectances and surface BRDF retrieval: A sensitivity study. IEEE Trans. Geosci. Remote Sens. 1999, 37, 724-738.

17. Van Zyl, J.J. The Shuttle Radar Topography Mission (SRTM): A breakthrough in remote sensing of topography. Acta Astronaut. 2001, 48, 559-565. [CrossRef]

18. Banks, A.C.; Hunt, S.E.; Gorroño, J.; Scanlon, T.; Woolliams, E.R.; Fox, N.P. A comparison of validation and vicarious calibration of high and medium resolution satellite-borne sensors using RadCalNet. In Proceedings of the Sensors, Systems, and NextGeneration Satellites XXI, Warsaw, Poland, 11-14 September 2017; Volume 10423, p. 104231A.

19. Anderson, N.J.; Czapla-Myers, J.S. Ground viewing radiometer characterization, implementation and calibration applications: A summary after two years of field deployment. In Earth Observing Systems XVIII; International Society for Optics and Photonics: Bellingham, WA, USA, 2013; Volume 8866, p. 88660N. [CrossRef]

20. Vescovi, F.D.; Lankester, T.; Coleman, E.; Ottavianelli, G. Harmonisation initiatives of Copernicus data quality control. Int. Arch. Photogramm. Remote Sens. Spat. Inf. Sci. 2015, 40, 713. [CrossRef]

21. Lenoble, J. (Ed.) Radiative Transfer in Scattering and Absorbing Atmospheres: Standard Computational Procedures; A Deepak: Hampton, VA, USA, 1985; Volume 300.

22. Ross, J. The Radiation Regime and Architecture of Plant Stands; Springer: Norwell, MA, USA, 1981; Volume 392, p. 1981.

23. Franch, B.; Vermote, E.; Skakun, S.; Roger, J.-C.; Masek, J.; Ju, J.; Villaescusa-Nadal, J.L.; Santamaria-Artigas, A. A method for Landsat and Sentinel 2 (HLS) BRDF normalization. Remote Sens. 2019, 11, 632. [CrossRef] 
24. Lyapustin, A.I.; Wang, Y.; Laszlo, I.; Hilker, T.; Hall, F.G.; Sellers, P.J.; Tuckera, C.J.; Korkin, S.V. Multi-angle implementation of atmospheric correction for MODIS (MAIAC): 3. Atmospheric correction. Remote Sens. Environ. 2012, 127, 385-393. [CrossRef]

25. Vermote, E.F.; Tanré, D.; Deuze, J.L.; Herman, M.; Morcette, J.J. Second simulation of the satellite signal in the solar spectrum, 6S: An overview. IEEE Trans. Geosci. Remote Sens. 1997, 35, 675-686. [CrossRef]

26. Samain, O.; Roujean, J.L.; Geiger, B. Use of a Kalman filter for the retrieval of surface BRDF coefficients with a time-evolving model based on the ECOCLIMAP land cover classification. Remote Sens. Environ. 2008, 112, 1337-1346. [CrossRef]

27. Stark, P.B.; Parker, R.L. Bounded-variable least-squares: An algorithm and applications. Comput. Stat. 1995, 10, 129-142.

28. Einicke, G.A.; White, L.B. Robust extended Kalman filtering. IEEE Trans. Signal Process. 1999, 47, 2596-2599. [CrossRef]

29. Niro, F.; Goryl, P.; Dransfeld, S.; Boccia, V.; Gascon, F.; Adams, J.; Themann, B.; Scifoni, S.; Doxani, G. European Space Agency (ESA) Calibration/Validation Strategy for Optical Land-Imaging Satellites and Pathway towards Interoperability. Remote Sens. 2021, 13, 3003. [CrossRef] 